\title{
Hidden Optimal Principle in Quantum Mechanics and Quantum Chemistry
}

\author{
Edward H. Jime nez $z^{1,2}$ \\ ${ }^{1}$ LaMUSE, Laboratoire de Mathématiques de l'Université de Saint-Étienne, France \\ ${ }^{2}$ Research and Development Department, EPPetroecuador, Quito, Ecuador \\ ejimenezecu@yahoo.com
}

\begin{abstract}
Quantum Mechanic axio ms are the result of the microscopic cooperative equilibriu mamong sy mmetric players (quantum object and human subject). On the other hand, we introduce Nash's equilibrium in Hilbert space, which has two characteristics: it is a fixed point and it maximizes a utility function. Moreover, evolution and selfadjoint operators have interesting properties which allow us to study steady state in Evolutionary Game Theory. Also, we present cooperative games in complex systems language. The concept of cooperation is important in game theory but is some what subtle. The term cooperate means "to act together, with a common purpose". Incentive compatibility is equivalent to synergy principle, which appears naturally (to add or to multiply utilities, ec[34]). Finally, wein the relevant results not only proved the main theorems ofQuantum Mechanics, Quantum Chemistry and its applications to salt-water but also we resolved some main questions such us: Do hydrogen-bonded networks, in which tunneling plays an important role, exist?, How cooperation and entropy affect water cluster equilibria?. Can salt $(\mathrm{ClNa})$ play an Nobel role in photo-catalysis? The answers are: tunnel effect it is possible in salt water but not in water, because $\mathrm{ClNa}$, visib le light or electricity incentive to produce catalysis or photo-catalysis. Also, If hydrogen-bonded networks and water cluster size increases comple xity of an global equilibrium, then tunnel effect appears as an local equilibrium.
\end{abstract}

Keywords Econophysics, Optimal Principle, Cooperation, Nash Equilibriu m, Hilbertspace, Evolution Operator, Tunnel Effect

\section{Introduction}

We will use a systemic perspective which is authorized by research methodology of complex systems[2],[3], in order to present a vision that integrates quantum computing concepts [7] and game theory elements to study cooperative games. The angular stone that unifies these two approaches is classic information theory. However, there is a narrow and formal bridge to carry out these tasks denominated quantum probability. More easily, we can understand quantum probability as the Hilbert space of random variables with finite second mo ment, see[13],[16].

First, approaches related to cooperation appear. After that we carry out a revision of existent literature, such us communication, correlation, entanglement, dependence and common objectives, see[12]. Cooperative game theory enriched by Nash's product focus approaches common objective topic (resolution of common problems) using bargaining roads which allow us to discover and implement equilibria. Concepts such us correlation, entanglement and

* Corresponding author:

ejimenezecu@y ahoo.com(Edward H. Jimenez)

Published online at http://journal.sapub.org/ijtmp

Copyright (C) 2012 Scientific \& Academic Publishing. All Rights Reserved dependence are complementary and they are strongly related intuitively. Consequently, we will establish its limits and functional dependences. Finally,[1] and[14] show that coordination and communication concepts can replace correlation (entanglement or dependence), see[5].

Second, this work is an example how the analyses of game theory can exp lain the paradox of Einstein-PodolskiRosen (hereafter: ERP) satisfactorily. Nevertheless, Anton Zeilinger, an outstanding researcher in quantum computing, has already expressed that interaction between two particles inentanglement does not have theoretical repercussions in Einstein's relativity theory, see[4],[6].

Third, the main theorems of this paper allow us to obtain Nash's equilibrium in Hilbert space, which has two properties: it is a fixed point and maximizes a utility function

$<\mathrm{p}^{\max }|\mathrm{A}| \mathrm{p}^{\max }>$ where $\mid \mathrm{p}^{\max }>=\left(\left|\mathrm{x}_{1}^{\max }\right|^{2} \ldots\left|\mathrm{x}_{\mathrm{n}}^{\max }\right|^{2}\right)^{*}$. InHilbert spaces, the norm of a selfadjoint operator (utility matrix: A) permits us to obtain the fixed pointsf $(|x\rangle)=$ $\mathrm{A} / \lambda|\mathrm{x}>=| x>$, where $\|\mathrm{A}\|=\max _{\|x\|=1}<\mathrm{x}|\mathrm{A}| \mathrm{x}>=$ $\lambda_{\max }$. A symmetrical matrix is a peculiar case of a selfadjoint operator, which has the advantage of being very usefull in bimatrix symmetrical games. A selfadjoint operator allows us to introduce utilities $\mathrm{a}_{\mathrm{ij}} \in \mathbb{C}$ defined on complex nu mbers. 
To sum up, Quantum Mechanic axioms are the result of the microscopic cooperative equilibrium among symmetrical players (quantum-object and human-subject). Also, properties of selfadjoint and evolution operators here proved, can be applied to two-player symmetric games. Moreover, this work is an application of econophysics in Quantum Mechanics, see[8], [9].

This paper is organized as follows. The first section is a revision of the existent literature about cooperation, entanglement and the Hilbert space of random variables. In the second section, we show the main theorems of complex games and its relationship with entanglement and ERP paradox.

In the third section we can see Nash's equilibrium in Hilbert spaces, evolution operator and steady state. The fourth section is the conclusion of this research.

\section{Cooperation in Economics and Quantum Mechanics}

We can denote any strategic-form game $\Gamma$ as $\Gamma=$ $\left(\mathrm{N},\left(\mathrm{S}_{\mathrm{j}}\right)_{\mathrm{j} \in \mathrm{N}},\left(\mathrm{u}_{\mathrm{j}}\right)_{\mathrm{j} \in \mathrm{N}}\right)$, where $\mathrm{N}$ is the set of players, $\mathrm{S}_{\mathrm{j}}$ is the set of strategies for player $\mathrm{j}$; and $\mathrm{u}_{\mathrm{j}}: \mathrm{S} \rightarrow \mathbb{R}$ is the utility payoff for player $j$. Here $S=\prod_{j \in N} S_{j}$ denotes the set of all possible combinations or profiles of strategies that may be chosen by the various players.

In general, a randomized strategy is any probability distribution over a set of strategies. We may denote such a randomized strategy in general by $\sigma=\left(\sigma(\mathrm{s})_{\mathrm{s} \in \mathrm{S}}\right)$, where $\sigma(S) \in \Delta(S)$ represents the probability of choosing $\mathrm{s}$, $\mathrm{u}_{\mathrm{j}}=\sum_{\mathrm{s} \in \mathrm{S}} \sigma(\mathrm{s}) \mathrm{u}(\mathrm{s}), \sigma(\mathrm{s})=\prod_{\mathrm{j} \in \mathrm{N}} \sigma_{\mathrm{j}}\left(\mathrm{s}_{\mathrm{j}}\right), \Delta(\mathrm{S})=\Delta\left(\prod_{\mathrm{j} \in \mathrm{N}} \mathrm{S}_{\mathrm{j}}\right)$ in correlated strategies and $\Delta(\mathrm{S})=\Delta\left(\prod_{\mathrm{j} \in \mathrm{N}} \mathrm{S}_{\mathrm{j}}\right)$, in non-correlated strategies. Note that in general $\Delta\left(\prod_{\mathrm{j} \in \mathrm{N}} \mathrm{S}_{\mathrm{j}}\right) \neq$ $\prod_{\mathrm{j} \in \mathrm{N}}\left(\Delta \mathrm{S}_{\mathrm{j}}\right)$.

The concept of cooperation is important in game theory but is somewhat subtle. The term cooperate means "to act together, with a common purpose".

The common purpose can be explicit, when players add or multiply energy or utility (synergy principle). Complex game theorem shows us that if we cooperate (to add or to multiply utility), then the results are correlated strategies and actions in order to maximize $\left(\sum_{j=1}^{n} u_{j}\right)$ or $\left(\prod_{j=1}^{n} u_{j}\right)$.

Definition 1.Complex-Game. We may define a cooperative transformation to be any mapping $\Psi$, such that if $\Gamma$ is a simple-game in strategic-form, then $\Psi(\Gamma)$ is another complex-game in strategic-form.

Entanglement concept and dependence are related. Entanglement is a general physical concept; nevertheless, sometimes it is used in correspondence with mathematical correlation. On the other hand, dependence is a mathematical concept which is more general than correlation because if two stochas tic variables $\xi$ and $\eta$ are uncorrelated $(\rho(\xi, \eta)=0)$, it does not follow that they are independent, see[16].

Definition 2.Dependent Strategy. A dependent (correlated) strategy for $\mathrm{N}$ players is any classic probability distribution in $(\mathrm{S})$, where $\sigma(\mathrm{s}) \neq \prod_{\mathrm{j} \in \mathrm{N}} \sigma_{\mathrm{j}}\left(\mathrm{s}_{\mathrm{j}}\right), \sigma(\mathrm{s}) \in \Delta(\mathrm{S})$, and $\Delta(\mathrm{S})=$ $\Delta\left(\prod_{\mathrm{j} \in \mathrm{N}} \mathrm{S}_{\mathrm{j}}\right)$. Note $\Delta\left(\prod_{\mathrm{j} \in \mathrm{N}} \mathrm{S}_{\mathrm{j}}\right) \neq \prod_{\mathrm{j} \in \mathrm{N}} \Delta\left(\mathrm{S}_{\mathrm{j}}\right)$.

Theorem 1. If a complex-game (cooperative game) is built such us $(u)=\sum_{j=1}^{n} u_{j}$, or $\varphi(u)=\prod_{j=1}^{n} u_{j}$, then density functions of $\varphi(\mathrm{u})$ are respectively $\mathrm{dF}_{\mathrm{z}}(\mathrm{z})=\int_{\mathbb{R}^{\mathrm{n}-1}} \mathrm{dF}(\mathrm{z}-$ $\left.\sum_{j=2}^{n} u_{j}, u_{2}, \ldots, u_{n}\right) \operatorname{ordF}_{z}(z)=\int_{R^{n-1}} \frac{d F\left(z / \prod_{j=2}^{n} u_{j}, u_{2}, \ldots, u_{n}\right)}{\prod_{j=2}^{n}\left|u_{j}\right|}$.

Proof. Let $U_{\mathrm{j} \in \mathrm{N}}$ be random variables with joint distribution function $\mathrm{F}_{\mathrm{U}_{1} \ldots \mathrm{U}_{\mathrm{n}}}$, and $\varphi(\mathrm{u})=\sum_{\mathrm{j}=1}^{\mathrm{N}} \mathrm{u}_{\mathrm{j}}$ be a Borel function. If we put $\mathrm{Z}=\varphi(\mathrm{u})$ we see at once that

$$
\begin{aligned}
& F_{Z}(\mathrm{z})=\int_{\left\{\mathrm{u}_{\mathrm{j}}: \sum_{\mathrm{j}=1}^{\mathrm{n}} \mathrm{u}_{\mathrm{j}} \leq \mathrm{z}\right\}} \mathrm{dF}\left(\mathrm{u}_{\mathrm{j}}\right) \\
& =\int_{\mathbb{R}^{\mathrm{n}}} \mathrm{I}_{\left\{\mathrm{u}_{\mathrm{j}}: \sum_{\mathrm{j}=1}^{\mathrm{n}} \mathrm{u}_{\mathrm{j}} \leq \mathrm{z}\right\}}\left(\mathrm{u}_{\mathrm{j}}\right) \mathrm{dF}\left(\mathrm{u}_{\mathrm{j}}\right) \\
& =\int_{\mathbb{R}^{\mathrm{n}-1}} \mathrm{I}_{\left\{\mathrm{u}_{\mathrm{j}}: \sum_{\mathrm{j}=1}^{\mathrm{n}} \mathrm{u}_{\mathrm{j}} \leq \mathrm{z}\right\}} \int_{\mathbb{R}}(\mathrm{u}) \mathrm{dF}\left(\mathrm{u}_{1}-\sum_{\mathrm{j}=2}^{\mathrm{n}} \mathrm{u}_{\mathrm{j}}, \mathrm{u}_{2}, \ldots, \mathrm{u}_{\mathrm{n}}\right)
\end{aligned}
$$

Note that incentive constraint (synergy principle) is respected because in the integral $z \geq \sum_{j=1}^{n} u_{j}$.

$$
\begin{aligned}
F_{Z}(z) & =\int_{\mathbb{R}^{n-1}} \int_{-\infty}^{z} d F\left(u_{1}-\sum_{j=2}^{n} u_{j}, u_{2}, \ldots, u_{n}\right) \\
& =\int_{-\infty}^{z} \int_{\mathbb{R}^{n-1}} d F\left(u_{1}-\sum_{j=2}^{n} u_{j}, u_{2}, \ldots, u_{n}\right)
\end{aligned}
$$

using the last equations we can write the density function of Z.

$$
F_{z}(z)=\int_{\mathbb{R}^{n-1}} d F\left(u_{1}-\sum_{j=2}^{n} u_{j}, u_{2}, \ldots, u_{n}\right.
$$

Let $U_{\mathrm{j} \in \mathrm{N}}$ be random variables with joint distribution functionsF $\mathrm{U}_{\mathrm{U}_{1} \ldots \mathrm{U}_{\mathrm{n}}}$, and $\varphi(\mathrm{u})=\prod_{\mathrm{j}=1}^{\mathrm{n}} \mathrm{u}_{\mathrm{j}}$ be a Borel function. The synergy principle is taken into account because in the integral $\prod_{\mathrm{j}=1}^{\mathrm{n}} \mathrm{u}_{\mathrm{j}} \leq \mathrm{z}$. If we put $=\varphi(\mathrm{u})$; we see at once that:

$$
\begin{aligned}
& F_{Z}(z)=\int_{\left\{u_{j}: \prod_{j=1}^{n} u_{j} \leq z\right\}} d F\left(u_{j}\right) \\
& =\int_{\mathbb{R}^{n}} I_{\left\{u_{j}: \prod_{j=1}^{n} u_{j} \leq z\right\}}(u) d F(u) \\
& =\int_{\mathbb{R}^{n-1}} \int_{\mathbb{R}^{\prime}} I_{\left\{u_{1}: u_{1 \leq z}\right\}}(u) \frac{d F\left(u_{1} / \prod_{j=2}^{n} u_{j}, u_{2}, \ldots, u_{n}\right)}{\prod_{j=2}^{n}\left|u_{j}\right|} \\
& F_{Z}(z)=\int_{\mathbb{R}^{n-1}} \int_{-\infty}^{z} \frac{d F\left(u_{1} / \prod_{j=2}^{n} u_{j}, u_{2}, \ldots, u_{n}\right)}{\prod_{j=2}^{n}\left|u_{j}\right|} \\
& =\int_{-\infty}^{Z} \int_{\mathbb{R}^{n-1}} \frac{d F\left(u_{1} / \prod_{j=2}^{n} u_{j}, u_{2}, \ldots, u_{n}\right)}{\prod_{j=2}^{n}\left|u_{j}\right|}
\end{aligned}
$$

using the last equations we can write the density function of Z.

$$
F_{z}(z)=\frac{d F\left(z / \prod_{j=2}^{n} u_{j}, u_{2}, \ldots, u_{n}\right)}{\prod_{j=2}^{n}\left|u_{j}\right|}
$$

\subsection{The Hilbert Space of Random Variables with Finite Second Moment}

1. An important role among Banachspaces $L^{p}, p \geq 1$, is played by the space $\mathrm{L}^{2}=\mathrm{L}^{2}(\Omega, \mathcal{F}, \mathrm{P})$, the space of (equivalence classes of) random variables with finite second moments, see[16], pag 260-280.

If $\xi$ and $\eta \in \mathrm{L}^{2}$, we put the inner product as

$$
(\xi, \eta)=<\xi \mid \eta>=E \xi \eta
$$

Also, the space $\mathrm{L}^{2}$ is complete with respect to the norm induced by the scalar product

$$
\|\xi\|=(\xi, \xi)^{1 / 2}
$$

2. Two random variables $\xi$ and $\eta \in \mathrm{L}^{2}$ are said to be 
orthogonal $(\xi \perp \eta)$ if $(\xi, \eta)=E \xi \eta=0$. In other words, they are uncorrelated $\operatorname{cov}(\xi, \eta)=0$, i.e.

$$
E \xi \eta=E \xi E \eta
$$

It follows that the properties of being orthogonal and of being uncorrelated coincide for random variables with zero mean values.

3. Let $M=\left\{\eta_{1}, \ldots, \eta_{n}\right\}$ be an orthonormal system and $\xi$ any random variable in $\mathrm{L}^{2}$. Consequently the best (in the mean-square sense) estimator for $\xi$ in terms of $\eta_{1}, \ldots, \eta_{n}$ is

$$
\hat{\xi}=\sum_{j=i}^{n}\left(\xi, \eta_{j}\right) \eta_{j}
$$

Hilbert space methods are extensively used in probability theory to study properties that depend only on the first two mo ments of random variables (" $\mathrm{L}^{2}$-Theory").

4. Let $\mathrm{H}_{\mathrm{m}_{1}}, \ldots, \mathrm{H}_{\mathrm{m}_{\mathrm{n}}}$ be Hilbert spaces with bases $\left\{\mathrm{al}_{1}^{\mathrm{j}}, \ldots, \mathrm{ad}_{\mathrm{m}_{\mathrm{j}}}^{\mathrm{j}}\right\}$. The tensor product of spaces $\mathrm{H}=$ $\mathrm{H}_{\mathrm{m}_{1}}, \ldots, \mathrm{H}_{\mathrm{m}_{\mathrm{n}}}$ is denoted by $\mathrm{H}=\mathrm{H}_{\mathrm{m}_{1}} \otimes \ldots \otimes \mathrm{H}_{\mathrm{m}_{\mathrm{n}}}$.

Using Dirac's notation, space $\mathrm{H}$ has ordered elements $\left(a_{j_{1}}^{1}, \ldots, a_{j_{n}}^{n}\right)=\left|a_{j_{1}}^{1}>\ldots\right| a_{j_{n}}^{1}>=\mid a_{j_{1}}^{1}, \ldots, a_{j_{n}}^{n}>$

as a basis, thus $\mathrm{H}$ has dimensionm $\mathrm{m}_{1} \mathrm{~m}_{2} * \ldots * \mathrm{~m}_{\mathrm{n}}$.

As in the case of classic and probabilistic systems, the basis states of the compound system $\mathrm{H}$ can be thought of $\left(a_{j_{1}}^{1}, \ldots, a_{j_{n}}^{n}\right)$ elements. It is natural to represent the general state of the compound system as

$$
\sum_{\mathrm{j}_{1}=1}^{\mathrm{m}_{1}} \ldots \sum_{\mathrm{j}_{\mathrm{n}}=1}^{\mathrm{m}_{\mathrm{n}}} \alpha_{\mathrm{j}_{1} \ldots \mathrm{j}_{\mathrm{n}}}\left(\mathrm{a}_{\mathrm{j}_{1}}^{1}, \ldots, \mathrm{a}_{\mathrm{j}_{\mathrm{m}}}^{\mathrm{j}}\right)
$$

where

$$
\sum_{\mathrm{j}_{1}=1}^{\mathrm{m}_{1}} \ldots \sum_{\mathrm{j}_{\mathrm{n}}=1}^{\mathrm{m}_{\mathrm{n}}}\left|\alpha_{\mathrm{j}_{1} \ldots \mathrm{j}_{\mathrm{n}}}\right|^{2}=1 \text {. }
$$

decomposable system if:

$$
\begin{gathered}
\sum_{\mathrm{j}_{1}=1}^{\mathrm{m}_{1} \ldots} \sum_{\mathrm{j}_{\mathrm{n}}=1}^{\mathrm{m}_{\mathrm{n}}} \alpha_{\mathrm{j}_{1} \ldots \mathrm{j}_{\mathrm{n}}}\left(\mathrm{a}_{\mathrm{j}_{1}}^{1}, \ldots, \mathrm{a}_{\mathrm{j}_{\mathrm{n}}}^{\mathrm{n}}\right) \\
=\sum_{\mathrm{j}_{1}=1}^{\mathrm{m}_{\mathrm{n}}} \alpha_{\mathrm{j}_{1}} \alpha_{\mathrm{j}_{\mathrm{n}}}\left|\mathrm{a}_{\mathrm{j}_{1}}^{1}>\ldots \sum_{\mathrm{j}_{\mathrm{n}}=1}^{\mathrm{m}_{1}}\right| \mathrm{al}_{\mathrm{j}_{\mathrm{n}}}^{\mathrm{n}}> \\
=\left(\sum_{\mathrm{j}_{1}=1}^{\mathrm{m}_{1}} \alpha_{\mathrm{j}_{1}} \mid \mathrm{al}_{\mathrm{j}_{1}}^{1}>\right) \ldots\left(\sum_{\mathrm{j}_{\mathrm{n}}=1}^{\mathrm{m}_{\mathrm{n}}} \alpha_{\mathrm{j}_{\mathrm{n}}} \mid \mathrm{a}_{\mathrm{j}_{\mathrm{n}}}^{1}>\right)
\end{gathered}
$$

Definition 3. We say that the compound system is entangled if it is not decomposable.

\subsection{Correlated Game and ERP Paradox}

In Quantum Information Theory, the correlated equilibria in two-player games mean that the associated probabilities of each-player strategies are dependent. Entanglement, according to the Austrian physicist Erwin Shrödinger, which is the essence of Quantum Mechanics, has been known for a long time now to be the source of a number of paradoxical and counterintuitive phenomena. Of those, the most remarkable one is the so-called non-locality, which is at the heart of the Einstein-Podolsky-Rosen paradox (ERP) see[12]pag 12. Einstein, Podolsky and Rosen consider a quantum system consisting of two particles separated by a great distance.

"EPR sugges ts that measure ment on partic le 1 cannot have any actual influence on particle 2 (locality condition); thus the property of particle 2 must be independent of the measurement performed on particle 1".

Nevertheless, the experiments verified that two particles in the EPR case are always part of one quantum system and thus measurement on one particle changes the possible predictions that can be made for the whole system and therefore for the other partic le[5].

Moreover, the essence of the EPR argument is as follows. EPR was interested in what termed "elements of reality". Their belief was that any such element of reality must be represented in any complete physical theory. The goal of the argument was to show that Quantum Mechanics is not a complete physical theory, by identifying elements of reality that were not included in Quantum Mechanics. The way they attempted to do this was by introducing what they claimed was a sufficient condition for a physical property to be an element of reality, namely, that it be possible to predict with certainty the value that property will have, immediately before measurement, see[15].

To illustrate, applying "revelation principle" for strategic-form games, the EPR paradox is solved automatically, because this principle demonstrates that all correlated game (entanglement) can be replaced by a communication game, see[14].

"...any equilibrium of any communication game can be generated from a strategic-form game $\Gamma$ by adding a system for preplay commun ication mustbe equivalent to a correlated equilibrium..."

Thus, according to[1], "a correlated equilibrium is any correlated strategy for the players in $\Gamma$ that could be self-enforcingly implemented with the help of a mediator who can make nonbinding confidential recommendations to each player."

Finally, nature is optimal and does not need to have replied or equivalent properties simultaneously such as (entanglement $\wedge$ communication).Therefore, we are in the presence of an exclusive-or (entanglement $\vee$ communicatio n).

If in a theoretical or experimental way we can demonstrate that a system ( $n$-player game) is entanglement, then ifis not necessary to speak of co mmunication. In short, game theory helps to resolve EPR paradox.

\section{Nash's Equilibrium in Hilbert Space}

The modern description of Quantum Mechanics is profoundly based on linear mappings. Here, we represent the features of linear mappings which are most essential for Quantum Mechanics. Because we mainly concentrate on finite level quantum systems, the vector spaces that are treated hereafter will be assumed to have a fin ite dimension, unless exp licitly stated otherwise, see[7].

Let us begin with some terminology: a linear mapping $\mathrm{H} \rightarrow \mathrm{H}$ is called an operator. The set of operators on $\mathrm{H}$ is denoted L(H): For an operator T; we define the norm of the operator by

$$
\|\mathrm{T}\|=\sup _{\|\mathrm{x}\|=1}\|\mathrm{Tx}\|=\lambda_{\max }
$$

A nonzero vector $x \in H$ is an eigenvector of $T$ belonging to eigenvalue $\lambda \in \mathbb{C}$ if $\mathrm{T} x=\lambda \mathrm{x}$. 
Remark 1 . With a fixed basis $\left\{\mathrm{e}_{1}, \ldots, \mathrm{e}_{\mathrm{n}}\right\}$ of $\mathrm{H}$; any operator $\mathrm{T}$ can be represented as $\mathrm{n} \times \mathrm{n}$ matrix over the field of complex numbers. It is not difficult to see that the matrix representing the adjoint operator $\mathrm{T}^{*}$ is the transposed complex conjugate of the matrix representing $\mathrm{T}$.

Definition 4. An operator $\mathrm{T}$ is selfadjo int if $\mathrm{T}=\mathrm{T}^{*}$. An operator $\mathrm{T}$ is unitary if $\mathrm{T}=\mathrm{T}^{-1}$.

Lemma 1. A selfadjo int operator has real eigenvalues.

Proof.IfA $x=\lambda x$ then using braked notation where $x=\mid x>$ is a column vector and $\mathrm{x}=<\mathrm{x} \mid$ is a row vector.

$\lambda^{*}\langle x \mid x\rangle=\langle\lambda x \mid x\rangle=\langle A x \mid x\rangle=\lambda\langle x \mid x\rangle$

since $\mid \mathrm{x}>\neq 0$ as an eigenvector, it fo llows that $\lambda^{*}=\lambda$.

Lemma 2. The eigenvectors of selfadjoint operators belonging to distinct eigenvalues are orthogonal.

Proof. Assume that $\lambda \neq \lambda^{\prime}, A x=\lambda x$, and $A x^{\prime}=\lambda^{\prime} x^{\prime}$. Since $\lambda, \lambda^{\prime}$ are real by the previous lemma,

$\lambda^{\prime}\left\langle x^{\prime} \mid x\right\rangle=\left\langle A x^{\prime} \mid x\right\rangle=\left\langle x^{\prime} \mid A x\right\rangle=\lambda\left\langle x^{\prime} \mid x\right\rangle$ and therefore $\left\langle x^{\prime} \mid x\right\rangle=0$.

Theorem 2. The matrix of eigenvectors $\mathrm{X}$ is unitary $\mathrm{X}^{*}=$ $\mathrm{X}^{-1}$ which permits us to write the spectral theorem $\mathrm{A}=$ $\mathrm{X} \Lambda \mathrm{X}^{-1}=\sum \lambda_{\mathrm{j}}\left|\mathrm{X}^{(\mathrm{j})}><\mathrm{x}^{(\mathrm{j})}\right|$.

Proof.Assume that $A\left|x^{(j)}>=\lambda_{j}\right| x^{(j)}>$ where

$x^{(j)}=\mid x^{(j)}>=\left(\begin{array}{l}x_{1}^{(j)} \\ x_{n}^{(j)}\end{array}\right)$ represents a column vector and $\mathrm{X}=\left(\mathrm{x}^{(1)} \ldots \mathrm{x}^{(\mathrm{n})}\right)$ is a $\mathrm{n} \times \mathrm{n}-$ matrix. Also it is easy to see that $\mathrm{AX}=\Lambda \mathrm{X}$ where

$$
\Lambda=\left(\begin{array}{cccc}
\lambda_{1} & 0 & 0 & 0 \\
\cdots & \cdots & \cdots & \cdots \\
0 & 0 & \lambda_{\mathrm{j}} & 0 \\
0 & 0 & 0 & \lambda_{\mathrm{n}}
\end{array}\right)
$$

Using matrix properties and vector orthogonality $<$ $\mathrm{x}^{(\mathrm{i})}\left|\mathrm{x}^{(\mathrm{j})}\right\rangle=0$ when $\mathrm{i} \neq \mathrm{j}$ and $\left\langle\mathrm{x}^{(\mathrm{j})} \mid \mathrm{x}^{(\mathrm{j})}\right\rangle=1$ for all $\mathrm{j}$.

$$
\mathrm{X} * \mathrm{X}=\left(\begin{array}{cccc}
\left\langle\mathrm{x}^{(1)} \mid \mathrm{x}^{(1)}\right\rangle & 0 & 0 & 0 \\
\ldots & \ldots & \ldots & \ldots \\
0 & 0 & \left\langle\mathrm{x}^{(\mathrm{j})} \mid \mathrm{x}^{(\mathrm{j})}\right\rangle & 0 \\
0 & 0 & 0 & \left\langle\mathrm{x}^{(\mathrm{n})}\right| \mathrm{x}^{\left({ }^{(n)}\right\rangle}
\end{array}\right)
$$

Thus, we conclude that $X^{*}=X^{-1}$. Here the symbol $(*)$ means hermitian transpose. Finally, using $A X=\Lambda X$, it is possible to write the spectral theorem.

$$
\begin{gathered}
\mathbf{A}=\mathbf{X} \Lambda \mathbf{X}^{-1}=\mathbf{X} \Lambda \mathbf{X}^{-1}=\sum_{\mathbf{j}=\mathbf{1}}^{\mathrm{n}} \lambda_{\mathbf{j}}\left|\mathbf{x}^{(\mathrm{j})}><\mathbf{X}^{(\mathbf{j})}\right| \\
\boldsymbol{\Lambda}=\mathbf{X}^{-\mathbf{1}} \mathbf{A X}
\end{gathered}
$$

We should revise the following postulates with the purpose of finding a relationship between Nash's equilibrium and the eigenvalues of Quantum Mechanics operators.

First Postulate:At a fixed point $\mathrm{t}_{0}$, the state of a physical system is defined by specifying a $\operatorname{ket} \mid \mathbf{x}\left(\mathbf{t}_{\mathbf{0}}\right)>$ belonging to the Hilbert space (state space) $\mathrm{H}$.

Second Postulate:Every measurable physical quantity A is described by an operator A acting in the state space $\mathrm{H}$.

Third Postulate:The only possible result of the measurement of a physical quantity $\mathrm{A}$ is one of the eigenvalues of the corresponding observable A. A measurement of A always gives a real value, since A is by definition Hermitian (selfad joint operator).
A measurement process in Quantum Mechanics is the best example of strategic interaction between "human-subject and quantum-object". Therefore, we have a minimu m of two symmetric players when we carry out a measurement process of a physical variable.

Theorem 3.The norm of a selfadjoint operator. The maximum eigenvalue $\lambda_{\max }=\max _{\|x\|=1}\langle x|A| x\rangle$ represents the maximum expected value of the selfadjoint operator $\mathrm{A}$ and its eigenvector $\left|\mathrm{x}^{\max }\right\rangle$ is a fixed point

$$
\frac{\mathrm{A}}{\lambda_{\max }}\left|\mathrm{x}^{\max }>=\right| \mathrm{x}^{\max }>
$$

Proof.The problem to maximize has the next Lagrangian:

$$
\mathcal{L}=<\mathrm{x}|\mathrm{A}| \mathrm{x}>-\lambda(<x \mid x>-1)
$$

first order condition $\partial \mathcal{L} / \partial<\mathrm{x} \mid=0$ implies the equation of eigenvectors.

$$
\mathrm{A}|\mathrm{X}>-\lambda| \mathrm{X} \geq 0
$$

Let $\max \langle A\rangle_{\mathrm{H}}$ be the maximum expected value in a Hilbert space, using equations (20), (21), (22).

$$
\begin{aligned}
\max <A & >_{\mathrm{H}}=\max _{\|\mathrm{X}\|=1}<x|A| x> \\
& =\max _{\|\mathrm{X}\|=1}<x\left|\mathrm{XX}^{-1} \mathrm{AXX}^{-1}\right|_{\mathrm{X}}>(25)
\end{aligned}
$$$$
\max _{\|y\|=1}<y\left|\mathrm{X}^{-1} \mathrm{AX}\right| \mathrm{y}>=\max _{\|\mathrm{y}\|=1}<y|\Lambda| y>
$$

we can write $\langle y|=X\langle x|$, $|\mathrm{y}\rangle=\mathrm{X}^{*} \mid \mathrm{x}>$ and $<$ $y|\mathrm{y}\rangle=\langle x \mid \mathrm{x}\rangle=1$. Using these last equations we have

$$
\max <A>_{\mathrm{H}}=\max _{\|y\|=1} \sum_{\mathrm{j}=1}^{\mathrm{n}} \lambda_{\mathrm{j}}\left|y_{\mathrm{j}}\right|^{2}=\lambda_{\max }
$$

Another form in order to choose $\langle A\rangle_{\mathrm{H}}$ is using the orthogonal eigenvector $x_{\max }$ where

$$
\begin{aligned}
\mathrm{A} \mid \mathrm{x}^{\max }> & =\lambda_{\max } \mid \mathrm{x}^{\max }>. \\
\max <A> & >_{\mathrm{H}}=<\mathrm{x}^{\max }|\mathrm{A}| \mathrm{x}^{\max }> \\
& =\lambda_{\max }<\mathrm{x}^{\max } \mid \mathrm{x}^{\max }>=\lambda_{\max }(27)
\end{aligned}
$$

Lemma 3.Nash's equilibrium in Hilbert space. In mixed strategy Nash's equilibrium, the eigenvector which maximizes $\langle A\rangle_{\mathrm{H}}=\langle x|A| x\rangle$ also maximizes expected utility, where $\max _{\|\mathrm{x}\|=1} \mathrm{E}(\mathrm{u})=\max <A>_{\mathrm{H}}=<\mathrm{p}^{\max }|\mathrm{A}| \mathrm{p}^{\max }>$ and

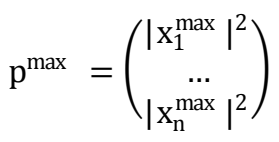

Proof. Let A be the matrix of utilities in a bimatrix game where expected utility is given by

$$
\mathrm{E}(\mathrm{u})=<A>_{\mathrm{H}}=<p|\mathrm{~A}| \mathrm{p}>\text { (29) }
$$

taking into account the properties of the eigenvector matrix $\mathrm{X}$; and of the orthogonal eigenvector $\|\mathrm{x}\|=1$ and equations (20), (21), (22).

$$
\begin{gathered}
\max _{\|\mathrm{X}\|=1}<A>_{\mathrm{H}}=\max _{\|\mathrm{X}\|=1}<p|\mathrm{~A}| \mathrm{p}> \\
=\max _{\|\mathrm{x}\|=1}<p\left|\mathrm{XX}^{-1} \mathrm{AXX}^{-1}\right| \mathrm{p}>(30) \\
=\max _{\|\mathrm{z}\|<1}<z\left|\mathrm{X}^{-1} \mathrm{AX}\right| \mathrm{z}>=\max _{\|\mathrm{z}\|<1}<z|\Lambda| z>
\end{gathered}
$$

we can write $\left.<z|=<p| X,\left|\mathrm{z}>=\mathrm{X}^{*}\right| \mathrm{p}\right\rangle$, where $\left\|_{\mathrm{z}}\right\|=\left\langle z|z>,\|\mathrm{p}\|=<p| p>\right.$ and $\left\|_{\mathrm{z}}\right\|=\|\mathrm{p}\|<1$. Using these last equations, we have mixed strategy Nash's equilibrium.

$$
\max <A>_{\mathrm{H}}=\max _{\|z\|} \sum_{\mathrm{j}=1}^{\mathrm{n}} \lambda_{\mathrm{j}}\left|\mathrm{z}_{\mathrm{j}}\right|^{2}=\sum_{\mathrm{j}=1}^{\mathrm{n}} \lambda_{\mathrm{j}}\left|\mathrm{z}_{\mathrm{j}}^{\max }\right|^{2}(31)
$$

It is evident that there is a vector $z^{\max }=\left(z_{1}^{\max }, \ldots, z_{n}^{\max }\right)^{*}$ where $\mathrm{A} \mid \mathrm{x}^{\max }>=$ $\lambda \operatorname{maxxmax}>$ and 


$$
\sum_{\mathrm{j}=1}^{\mathrm{n}} \lambda_{\mathrm{j}}\left|\mathrm{z}_{\mathrm{j}}^{\max }\right|^{2} \geq \sum_{\mathrm{j}=1}^{\mathrm{n}}\left|\mathrm{z}_{\mathrm{j}}\right|^{2} \text { for all }|\mathrm{z}>\neq| \mathrm{z}^{\max }>
$$

This last result follows

$$
\max <A>_{N}=\sum_{j=1}^{n} \lambda_{j}\left|z_{j}^{\max }\right|^{2}=<p^{\max }|A| p^{\max }>
$$

Moreover, in the case of a minimum, the problem can be resolved in a similar way. Finally, obtaining Nash's equilibrium not only requires fixed point theorem but also maximization of expected utility.

Example 1. Let A be a matrix of utilities of a bimatrix symmetrical game.

$$
\Lambda=\left(\begin{array}{ccccc}
1 & 4+2 i & -1 & -i & 1 \\
4-2 i & -3 & 0 & 1 & -2 \\
-1 & 0 & 4 & 1 & 6 \\
\mathrm{i} & 1 & 1 & 2 & 2 \\
1 & -2 & 6 & 2 & 5
\end{array}\right)
$$

Eigenvalues $\lambda_{i}$ are every one of the diagonal matrix elements of $\Lambda$.

$$
\left(\begin{array}{ccccc}
-6.8998 & 0 & 0 & 0 & 0 \\
0 & -0.95157 & 0 & 0 & 0 \\
0 & 0 & 1.4939 & 0 & 0 \\
0 & 0 & 0 & 4.1909 & 0 \\
0 & 0 & 0 & 0 & 11.167
\end{array}\right)
$$

Eigenvectors $\left|\mathrm{x}^{(\mathrm{j})}\right\rangle$ permit $\mathrm{us}$ to build the matrix $\mathrm{X}=\left(\mathrm{x}^{(1)} \mathrm{x}^{(2)} \mathrm{x}^{(3)} \mathrm{x}^{(4)} \mathrm{X}^{(5)}\right)$, where $\mathrm{AX}=\mathrm{X} \Lambda$ and $\mid \mathrm{p}^{(\mathrm{j})}>=$ $\left(\left|\mathrm{x}_{1}^{(\mathrm{j})}\right|^{2} \ldots\left|\mathrm{x}_{5}^{(\mathrm{j})}\right|^{2}\right)^{*}$.

$$
\begin{aligned}
& \left|\mathrm{x}^{(1)}\right\rangle=\left(\begin{array}{c}
0.26427+0.4346 \mathrm{i} \\
-0.64996-0.41449 \mathrm{i} \\
0.13389+0.13615 \mathrm{i} \\
0.15748+4.2475 * 10^{-2} \mathrm{i} \\
-0.22542-0.18198 \mathrm{i}
\end{array}\right) \\
& \left|x^{(2)}\right\rangle=\left(\begin{array}{c}
-0.07805-0.12936 i \\
0.31519-5.8172 * 10^{-2} i \\
0.70702+5.2601 * 10^{-2} i \\
1.6195 * 10^{-2}+0.08144 i \\
-0.53318-7.8377 * 10^{-2} i
\end{array}\right) \text {, } \\
& \left|x^{(3)}\right\rangle=\left(\begin{array}{c}
-0.15051-0.24753 i \\
-0.20756+0.10282 i \\
-8.1646 * 10^{-2}-0.14713 i \\
0.13679+0.89366 i \\
-1.3781 * 10^{-2}-0.12875 i
\end{array}\right), \\
& \left|x^{(4)}\right\rangle=\left(\begin{array}{c}
0.41293+0.67912 \mathrm{i} \\
0.39287+0.29085 \mathrm{i} \\
6.2676 * 10^{-2}-0.12865 \mathrm{i} \\
-3.2464 * 10^{-2}+0.31429 \mathrm{i} \\
7.6227 * 10^{-2}+5.6713 * 10^{-2} \mathrm{i}
\end{array}\right) \text {, } \\
& \left|x^{(5)}\right\rangle=\left(\begin{array}{c}
-2.8648 * 10^{-2}-4.7116 * 10^{-2} \mathrm{i} \\
-0.10129-1.2945 * 10^{-2} \mathrm{i} \\
0.64119+3.1297 * 10^{-2} \mathrm{i} \\
0.22202+5.1332 * 10^{-2} \mathrm{i} \\
0.72408+2.8675 * 10^{-2} \mathrm{i}
\end{array}\right)
\end{aligned}
$$

Using equations (25), (26), (27), (30), (31), and (32) by simple inspection, it is possible to verify that $\left\langle\mathrm{x}^{\max }|\mathrm{A}| \mathrm{x}^{\max }>=11.167=\lambda_{\max }>\lambda_{4}>\lambda_{3}>\lambda_{2}>\lambda_{1}\right.$.

On the other hand, we can see that $<\mathrm{p}^{\max }|\mathrm{A}| \mathrm{p}^{\max }>=$ $4.7836><\mathrm{p}^{2}|\mathrm{~A}| \mathrm{p}^{2}>=3.7295><\mathrm{p}^{3}|\mathrm{~A}| \mathrm{p}^{3}>=$
$1.5641><\mathrm{p}^{4}|\mathrm{~A}| \mathrm{p}^{4}>=1.4919><\mathrm{p}^{1}|\mathrm{~A}| \mathrm{p}^{1}>=$ 0.18383 in the same manner

$$
\begin{gathered}
\sum_{j=1}^{n} \lambda_{j}\left|z_{j}^{\max }\right|^{2}=4.7838>\sum_{j=1}^{n} \lambda_{j}\left|z_{j}^{(2)}\right|^{2} \\
=3.7296>\sum_{j=1}^{n} \lambda_{j}\left|z_{j}^{(3)}\right|^{2} \\
=1.5641>\sum_{j=1}^{n} \lambda_{j}\left|z_{j}^{(4)}\right|^{2} \\
=1.4919>\sum_{j=1}^{n} \lambda_{j}\left|z_{j}^{(1)}\right|^{2} \\
=0.1838
\end{gathered}
$$

where $\left|\mathbf{z}^{(\mathbf{j})}\right\rangle=\mathbf{X}^{*}\left|\mathbf{p}^{(\mathbf{j})}\right\rangle$.

\subsection{Cooperative Games in a Complex System Language}

We present cooperative games in the language of Complex Systems. Incentive compatibility is equivalent to synergy principle, which appears naturally (to add or to multiply utilities); on the other hand, coordination and communication have the same implications of correlation, see[14] page 256. Moreover, correlated strategies have a similar concept in Quantum Co mputing named entanglement. Correlation doesn't guarantee cooperation although this is equivalent to communication.

First, we need to replace the Nash Bargaining solution in two-person symmetric games. We define a bimatrix bargaining problem $\Gamma$ o consist of a pair $(F ; u)$ where $F$ is a closed convex subset of $\mathbb{R}^{2} ; \mathrm{u}=(\mathrm{u} ; \mathrm{u})$ is a vector in $\mathbb{R}^{2}$ and the set

$$
\mathbf{F} \cap\{(\mathbf{u}(\boldsymbol{\sigma}(\mathbf{s})), \mathbf{u}(\boldsymbol{\sigma}(\mathbf{s}))) \mid \boldsymbol{\sigma}(\mathbf{s}) \boldsymbol{\epsilon} \Delta(\mathbf{S})\}
$$

is nonempty and bounded. Here $F$ represents the set of feasible payoff allocations of the feasible set, and $\mathrm{V}$ represents the disagreement payoff allocation of the disagreement point. The utility is represented by

$$
\langle\mathbf{u}\rangle=\sum_{\mathbf{s} \in \mathbf{S}} \sigma(\mathbf{s}) \mathbf{u}(\mathbf{s})
$$

Second, there is a unique solution $\Phi(.,$.$) that satis fies the$ axio ms of Nash's bargaining solution. This solution function satis fies, for every two-pers on bargaining problem $(\mathbf{F}, \mathbf{v})$;

$$
\Phi(F, v) \epsilon \arg \max _{z \in F,} z \geq v(z-v)^{2}
$$

Quantum elements such us photons, electrons, subject-object have symmetrical physical properties and its two-player cooperative solution is the same for each one.

Finally, the special case of cooperation between symmetric particles or "human-subject andquantum-object" is when

$$
\mathrm{v}=0, \mathrm{u}=\langle\mathrm{p}|\mathrm{A}| \mathrm{p}\rangle,|\mathrm{p}\rangle=\left(\begin{array}{c}
\left|\mathrm{x}_{1}\right|^{2} \\
\ldots \\
\left.\mathrm{x}_{\mathrm{n}}\right|^{2}
\end{array}\right)
$$

and the Hermitian operator A can have complex or real values aij. Consequently, we can write $\Phi(\mathrm{F} ; 0)$ in Hilbert space

$$
\mathrm{z}=\Phi(\mathrm{F} .0)=\left\langle\mathrm{p}^{\max }|\mathrm{A}| \mathrm{p}^{\max }\right\rangle
$$




$$
\mathbf{A}\left|\mathbf{x}^{\max }\right\rangle=\lambda_{\max }\left|\mathbf{x}^{\max }\right\rangle
$$

\subsubsection{Evolution and Density Operators in Two-Player Symmetric Games}

Let us begin to write the expected utility $\mathrm{E}(\mathrm{u})=\overline{\mathrm{u}}$ of the operator (symmetric matrix) A that represents utilities. Here, we use Dirac's notation (braket), where |.) represents a column vector or ket and $\langle$.$| is a row vector or bra, and$ |. $\rangle=(\langle.|)^{*}$.

$$
\mathrm{E}(\mathrm{u})=\overline{\mathrm{u}}=\langle\mathrm{A}\rangle_{\mathrm{N}}=\langle\mathrm{p}|\mathrm{A}| \mathrm{p}\rangle
$$

the dynamic replicator equation in matrix form

$$
\frac{\mathrm{d}|\mathrm{p}\rangle}{\mathrm{dt}}=\left(\begin{array}{cccc}
\overline{\mathrm{u}}-\mathrm{u}_{1} & 0 & 0 & 0 \\
\cdots & \cdots & \cdots & \cdots \\
0 & 0 & \overline{\mathrm{u}}-\mathrm{u}_{1} 0 \\
0 & 0 & 0 \overline{\mathrm{u}}-\mathrm{u}_{1}
\end{array}\right)|\mathrm{p}\rangle
$$

let us write total derivative of expected value $\langle\mathrm{A}\rangle_{\mathrm{N}}$

$$
\frac{\mathrm{d}\langle\mathrm{A}\rangle_{\mathrm{N}}}{\mathrm{dt}}=\left(\frac{\mathrm{d}\langle\mathrm{p}|}{\mathrm{dt}}\right) \mathrm{A}|\mathrm{p}\rangle+\left\langle\mathrm{p}\left|\left(\frac{\partial \mathrm{A}}{\partial \mathrm{t}}\right)\right| \mathrm{p}\right\rangle+\langle\mathrm{p}| \mathrm{A}\left(\frac{\mathrm{d}|\mathrm{p}\rangle}{\mathrm{dt}}\right)
$$

usinga explicit form of $\frac{\mathrm{d}|\mathrm{p}\rangle}{\mathrm{dt}}=U|\mathrm{p}\rangle, \frac{\mathrm{d}\langle\mathrm{p}|}{\mathrm{dt}}=\langle\mathrm{p}| \mathrm{U}^{*}$ and given that $\mathrm{U}=\mathrm{U}^{*}$ we can write

$$
\begin{gathered}
\frac{\mathrm{d}\langle\mathrm{A}\rangle_{\mathrm{N}}}{\mathrm{dt}}=\langle\mathrm{p}|\mathrm{UA}| \mathrm{p}\rangle+\left\langle\mathrm{p}\left|\left(\frac{\partial \mathrm{A}}{\partial \mathrm{t}}\right)\right| \mathrm{p}\right\rangle+\langle\mathrm{p}|\mathrm{AU}| \mathrm{p}\rangle \\
\frac{d\langle A\rangle_{N}}{d t}=\langle p|U A+A U| p\rangle+\left\langle p\left|\left(\frac{\partial A}{\partial t}\right)\right| p\right\rangle
\end{gathered}
$$

using the nomenclature of an anticonmutator $\{\mathrm{U}, \mathrm{A}\}=$ $\mathrm{UA}+\mathrm{AU}$

$$
\frac{\mathrm{d}\langle\mathrm{A}\rangle_{\mathrm{N}}}{\mathrm{dt}}=\langle\{\mathrm{U}, \mathrm{A}\}\rangle+\left\langle\frac{\partial \mathrm{A}}{\partial \mathrm{t}}\right\rangle
$$

In a similar way, we can find evolution equation of density $\rho=|p\rangle\langle p|$

$$
\begin{gathered}
\frac{\mathrm{d} \rho}{\mathrm{dt}}=\left(\frac{\mathrm{d}|\mathrm{p}\rangle}{\mathrm{dt}}\right)\langle\mathrm{p}|+| \mathrm{p}\rangle\left(\frac{\mathrm{d}\langle\mathrm{p}|}{\mathrm{dt}}\right) \\
\frac{\mathrm{d} \rho}{\mathrm{dt}}=\mathrm{U}|\mathrm{p}\rangle\langle\mathrm{p}|+| \mathrm{p}\rangle\langle\mathrm{p}| \mathrm{U} \\
\frac{\mathrm{d} \rho}{\mathrm{dt}}=\{\mathrm{U}, \rho\}
\end{gathered}
$$

The evolution operator has integrated properties of symmetric games in strategic form and replicator dynamics in matrix form.

Steady State. Remember equations (20), (21), (22), specially $A X=X \Lambda$ and if we suppose that $\left\langle\frac{\partial \mathrm{A}}{\partial \mathrm{t}}\right\rangle=0$, then it follows that

$$
\frac{\mathrm{d}\langle\mathrm{A}\rangle_{\mathrm{N}}}{\mathrm{dt}}=\langle\{\mathrm{U}, \mathrm{A}\}\rangle
$$

By simple inspection, we can verify that the variable Uof the equation (37) can be written as $U=\langle A\rangle_{N} I-\Lambda$ replacing in equation (45)

$$
\frac{\mathrm{d}\langle\mathrm{A}\rangle_{\mathrm{N}}}{\mathrm{dt}}=\left\langle 2 \mathrm{~A}\langle\mathrm{~A}\rangle_{\mathrm{N}}-\{\mathrm{A}, \Lambda\}\right\rangle
$$

Simplifying equation (46)

$$
\frac{\mathrm{d}\langle\mathrm{A}\rangle_{\mathrm{N}}}{\mathrm{dt}}=2\langle\mathrm{~A}\rangle_{\mathrm{N}}^{2}-\langle\{\mathrm{A}, \Lambda\}\rangle
$$

Thus, if steady state is gotten when $\frac{d\langle A\rangle_{N}}{d t}=0$, then

$$
2\langle A\rangle_{N}^{2}-\langle P|\{A, \Lambda\}| P\rangle=0
$$

\subsection{Tunneling an Explicit Phenomenon of Optimal Cooper ation}

From viewpoint of classical mechanics, an electron cannot overcome a potential barrier higher than its energy. However, according to quantum mechanics, electrons are not defined by a precise position, but by a cloud of probability. This means that in some systems this probability cloud extends to the other side of a potential barrier. Therefore, the electron can cross the barrier, and for example, to generate electric current. This current is called the tunnel current and it is the control parameter that allows us to describe the topography of any surface.[5],[16].

A body with energy less than what is required to overcome or pass through a potential barrier can do it. There is a probability greater than zero, that a body goes through a potential barrier even if less energy is needed.

There is a probability g reater than zero, to find an object or phenomena associated due to its presence outside the potential barrier of energy higher than the same.

\section{Tunnel Effect in Hydrogen B onds}

Consider us the interaction of two water mo lecules, which form part of a larger complex, such as salt water clusters, where the $\mathrm{NaCl}$ is completely dissociated. If two water molecules form a hydrogen bond, then the hydrogen atom may or may not link to one of the oxygen atoms.[17],[18].

In our study we have families of clusters formed as follows: n-Na, n-Cl, n-Na-Cl, n-Cl-Na, n-H2O, with n, m, s element of the natural numbers.

$\mathrm{n}$ (water mo lecules)-m(sodium ato $\mathrm{ms}$ )-s(chlorine ato $\mathrm{ms}$ ).

Hydrogen and oxygen atoms of two water molecules are a distance below the threshold. On the other hand, a hydrogen appears uncoupled between two oxygen atoms, that is not linked specifically to any of the two oxygens and your extreme oscillatory motion penetrates the molecular orbitals of the two oxygen atoms, then we have a potential or feasible tunnel effect.

When hydrogen atom penetrates the orbital of one of the oxygen atoms, evidently out of the potential barrier of the other oxygen atom and vice versa. This implies that is constantly out of one of the potential barriers or put in another way: there is a probability greater than zero, that an atom leaves a potential barrier having a kinetic energy less than the height of the potential barrier.

Theorem.Tunnel effect is a phenomenon that emerges from external incitement such us catalysts presence and when the interior cooperation is maximum.

Proof.- Let it be a complex system of $\mathrm{n}$ players (quantum objects) $i=1, \ldots, n$, with a total energy E, which is the sum of each of energies Ei. Given a set of thresholds ui, which correspond one to one to each player i, where $\mathrm{Ei}<\mathrm{ui}$. An infinitesimal change in the total system energy $\mathrm{dE} / \mathrm{dt}$, can cause a considerable variation in a specific energy of one players i, $((\Delta \mathrm{Ei}) /(\Delta \mathrm{t}))$, which exceeds the respective threshold that player ui/ $\Delta \mathrm{t}$.

$$
((\mathrm{dE}) /(\mathrm{dt}))=((\Delta \mathrm{Ei}) /(\Delta \mathrm{t}))>((\mathrm{ui}) /(\Delta \mathrm{t})) .
$$

\subsubsection{Results}

First, according to our interpretation, the second law of thermodynamics is fulfilled, as it explicitly states that "given 
entropy, system energy is minimal since given energy, system entropy is maximum." We also know that in a state of equilibrium, the values taken by the characteristic parameters of a closed thermodynamic system are such that maximize the value of a certain magnitude called entropy. In this case, we are analyzing two related systems, which change entropy and energy value, this evolution cannot be explained by the second law of thermodynamics but can be explained by Theory of Quantum Games.

Second, following Game Theory and the Theory of Quantum Games, we say the optimal evolution of a complex system minimizes the entropy. Figure 1, Figure 2 and Figure 3 .

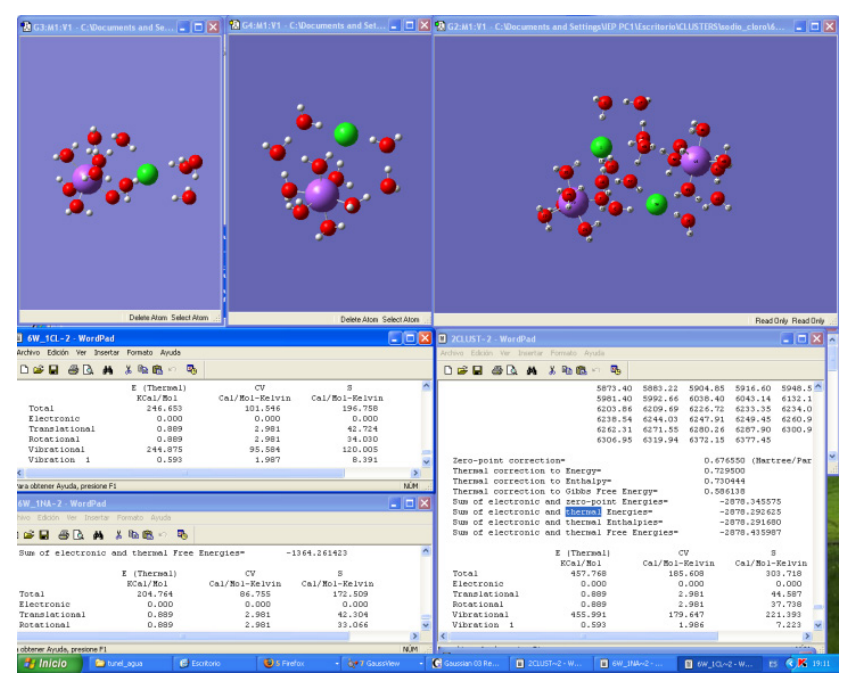

Figure 1. Cooperation and Minimum Entropy. We can observe, the left side cluster, where there are one sodium atom, one Chloride atom, and twelve water molecules, which are in equilibrium. That is, the balance of forces is zero and the energy of the system is minimal. The intermediate cluster consists of a sodium at om, a Chlorine atom and ten water molecules, also it is in equilibrium. The cluster on the right is the union of the previous clusters. Meanwhile also the right cluster is in equilibrium. The question is what happens to the value of entropy and energy when you bind left cluster on intermediate cluster. Just note that the resulting entropy of joining two clusters is less than the sum of individual entropies. We can verify that 303,718 Cal / Mol-Kelvin < 369.267 Cal / Mol-Kelvin. On the side of the energy we observe to increases: $457.768 \mathrm{Kcal}>451.420 \mathrm{Kcal}$. Entropy system is decreasing and energy is increasing. What Happens with second law of Thermodynamics with

Third, on the other hand, the cooperative equilibrium, maximize an objective function called utility, which is exactly equivalent to the energy of a thermodynamic system. In this particular example, we see that in both cases, entropy is minimized but not maximized energy, there is cooperation in the oxygen atoms that are the same type, whereas when there are atoms of different type converge to an equilibrium but not necessarily in a cooperative manner. Figure 2, Figure 3.

Fourth, using the analogy in question, we demonstrate experimentally and with the help of Quantum Chemistry that clusters of molecules that interact cooperatively, minimize entropy and maximize energy, which is the fundamental assumption of the Quantum Theory of Cooperative Games, which is the cornerstone of our discussion of tunneling in salt water and its future use. Figure 4.

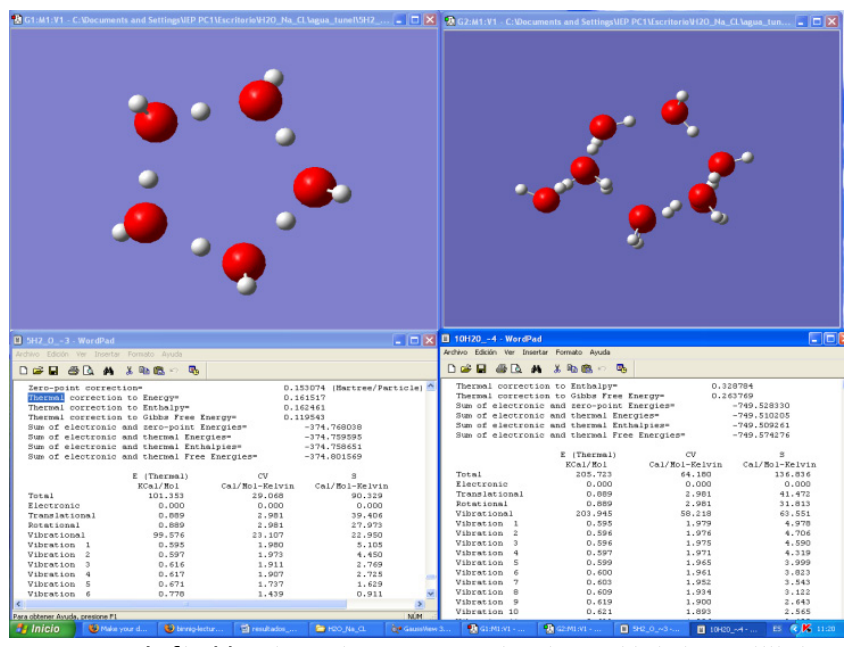

Figure 2. Left side cluster has $5 \mathrm{H}_{2} \mathrm{O}$ molecules and it is in equilibrium. That is, the balance of forces is zero and the energy of the system is minimal. Right side cluster is composed by ten water molecules. Meanwhile also the right cluster is in equilibrium. It is easy to see that entropy resulting from joiningtwo identical clusters is less than the sum of individual entropies. We can verify that $136,836 \mathrm{Cal} / \mathrm{Mol}-\mathrm{Kelvin}<(90.329+90.329) \mathrm{Cal} /$ Mol-Kelvin. On the side of energy, we could verify that energy increases: $205.723 \mathrm{Kcal}>(101.355+101,355) \mathrm{kcal}$. Again, entropy decreases and energy increases, what happens with the second law of thermodynamics

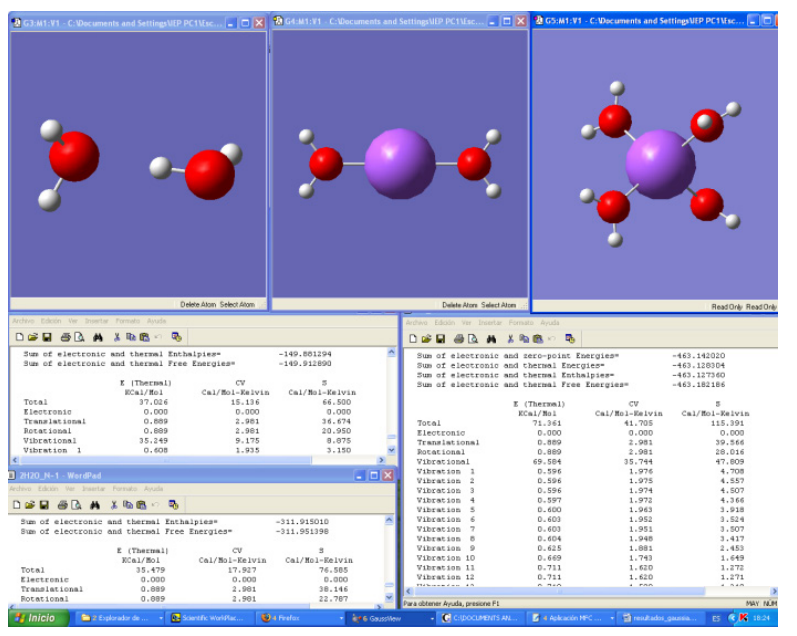

Figure 3. In left side cluster where there are two water molecules, it is in equilibrium. The intermediate cluster has a sodium atom and two water molecules, also it is optimized. Right side cluster is formed by union of left cluster and intermediate cluster. Meanwhile also right cluster is in equilibrium, according to four Gaussian criteria: Maximum Force, RMS Force, Displacement and RMS Maximum Displacement. It is easy to see that the resulting entropy of merging two clusters, left and middle is less than the sum of individual entropies. We can verify that $115.391 \mathrm{Cal} /$ Mol-Kelvin $<(66.585+76,585)$ Cal / Mol-Kelvin. On the side of energy, we could verify that energy decreases: $71.361 \mathrm{Kcal}<72.505 \mathrm{Kcal}$. The entropy and energy decrease. What happens to the second law of thermodynamics

Fifth, it is evident that pure water clustering tunnel effects not occur due to the non-presence of a NaCl-type catalyst. Figure 2.

Finally, theory of cooperative games makes clear that despite obtaining an invariant represented by entropy 
minimization, it is required energy maximization. Figure 4and Figure 8.

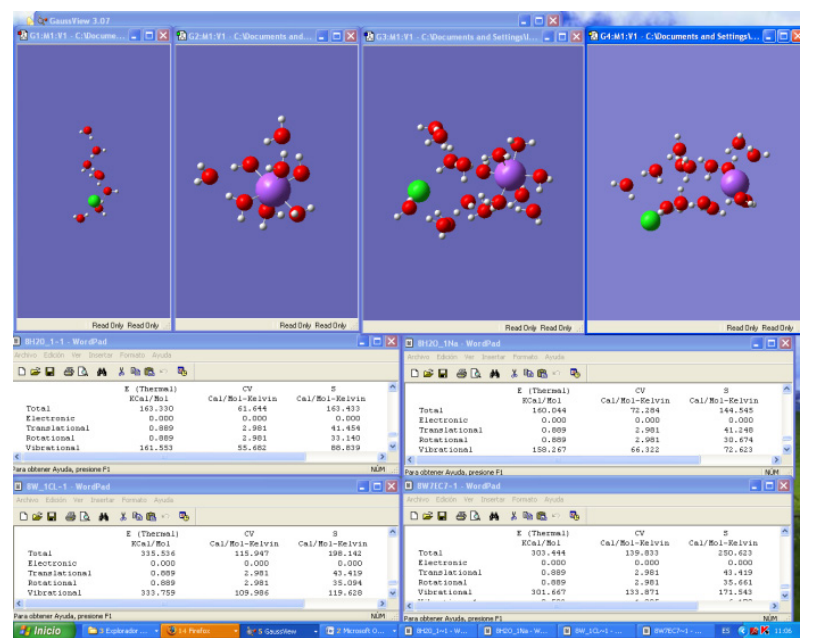

Figure 4. Cluster three is the union of cluster number one and cluster numberthree, and each one is in equilibrium. Cluster four is in equilibrium and observing its vibration frequency spectrum, it is clear that they have a large scatter in data, that otherwise have high statistical entropy. Physical entropy value of cluster three is minimal compared to cluster four value (198.42 Cal / Mol-Kelvin <250.623 Cal/Mol-Kelvin), while its energy is maximum, respect ively $(335.536 \mathrm{Kcal}>303.44 \mathrm{kcal})$

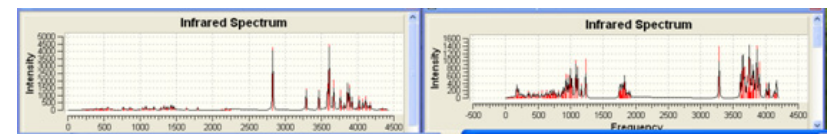

Figure 5. The left spectrum corresponds to third cluster in Figure 4, while the second spectrum corresponds to cluster four. Wenote in the graph, the spectral line $2825.32(1 / \mathrm{cm})$ corresponding to a frequency of tunneling, which disappears in the cluster number four

\begin{tabular}{|r|l|l|l|l|}
\hline $\mathbf{N}$ & O2 in Air & $\begin{array}{l}\text { Salt Water } \\
\text { Splitting }\end{array}$ & O2 & H2 \\
\hline $\mathbf{1}$ & 20,64 & 20,64 & & \\
\hline $\mathbf{2}$ & 20,64 & 20,65 & 0,01 & 0,02 \\
\hline $\mathbf{3}$ & 20,64 & 20,66 & 0,02 & 0,04 \\
\hline $\mathbf{4}$ & 20,64 & 20,67 & 0,03 & 0,06 \\
\hline $\mathbf{5}$ & 20,64 & 20,7 & 0,06 & 0,12 \\
\hline $\mathbf{6}$ & 20,64 & 20,69 & 0,05 & 0,1 \\
\hline $\mathbf{7}$ & 20,64 & 20,68 & 0,04 & 0,08 \\
\hline
\end{tabular}

Figure 6. Using a equipment to measure $\mathrm{O} 2$ concentration (AGS-688 y EGA-688 de BRAINBEE), we can verify presence of hydrogen because, when we do photo splitting salt water, appears oxygen and hydrogen simultaneously. In this figure we can see that $\mathrm{O} 2$ concentration increase with time, in reference with $\mathrm{O} 2$ base concentration equal to $20,64 \%$ and salt concentration $30 \% \mathrm{wt}$

The presence of a positive catalyst lowers the energy of the system, allowing it to develop a reaction with less energy and faster. Increasing effectiveness and reaction output power.

\section{Theoretical Results.}

One player (molecule, quantum element, water) which verifies Nash's equilibria is intelligent and optimize one utility function. That is the case of salt water.

Each physical variable is represented by anHermitian operator whose norm allows us to obtain mixed strategy Nash's equilibrium in Hilbert space.

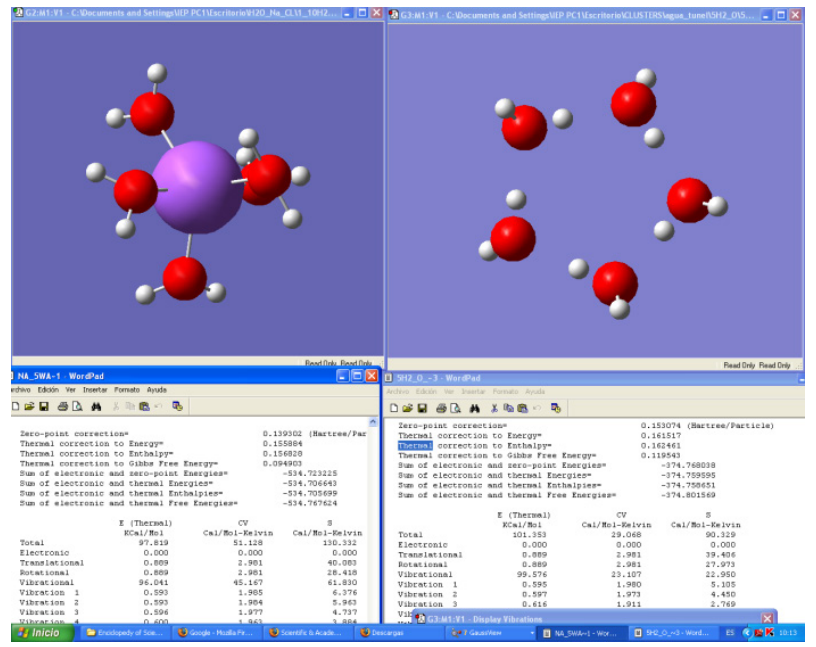

Figure 7. Differences of energy and entropy in the presence of a catalyst, as the case of sodium. The inner energy of the system without catalyst is lower, while the entropy is higher, which is logical, since the mere presence of the catalyst increases entropy

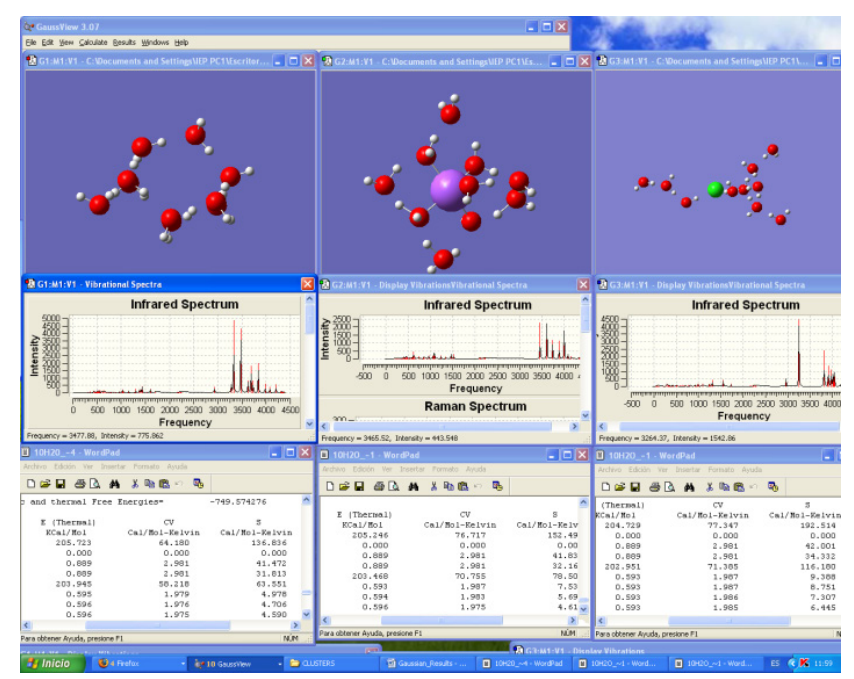

Figure 8. Variation of internal energy by presence of catalyst. The graphs left, right and middle explain that the internal energy is less when there is no catalyst (left graph). In the case of entropy, we know that it is minimal when there is no catalyst. To sum up, in a system it is verified that the entropy is minimal and the energy is maximum when there is no catalyst

By virtue of the theorems demonstrated in this paper, properties of Hermitian operators can only be used in symmetric games, which can be represented by complex matrix.

Mixed strategy Nash's equilibrium in a bimatrix symmetric game represents a cooperative solution when exist correlation exc lusive-or communication.

The idea of isolated physical systems has its explanation, the simplification of variables and relationships among the parts. On the contrary, complex systems analyze entirety, synergy and interactions as the cause of a common objective denominated cooperation.

The measurement process in Quantum Mechanics and Photocatalysis in Quantum Chemistry is the best example of 
strategic interaction between "quantum-subject (catalysts) and quantum-object (water) ." Therefore, we have a minimum of two players when we carry out a measurement of a physical variable such us energy, frequency, symmetry, and so on.

Lemma named Nash's equilibrium in Hilbert space explains that In mixed strategy Nash's equilibrium, the eigenvector which maximizes $<\mathrm{A}\rangle_{\mathrm{H}}=<\mathrm{x}|\mathrm{A}| \mathrm{X}>$ also maximizes expected utility, where: $\operatorname{maxE}(\mathrm{u})=\max <\mathrm{A}>_{\mathrm{N}}$ $=<\mathbf{p}^{\mathbf{m a x}}|\mathrm{A}| \mathbf{p}^{\mathbf{m a x}}>$. To use an analogy between utility and energy is explained in Jiménez, E.H (2003a, 2003b).

\section{Experimental Results.}

The presence of a positive catalyst lowers the energy of the system, allowing it to develop a reaction with less energy and faster. Increasing effectiveness and reaction output power. Figure 8.

In Figure 4, we can verify that standard deviation of spectrum has a relationship with system entropy.

Simple Quantum Catalysis does not verifies Nash's equilibria (minimum entropy and maximum energy). Figure 8,9 .We can see cluster quantum cooperation in figures 1,2 , 4.

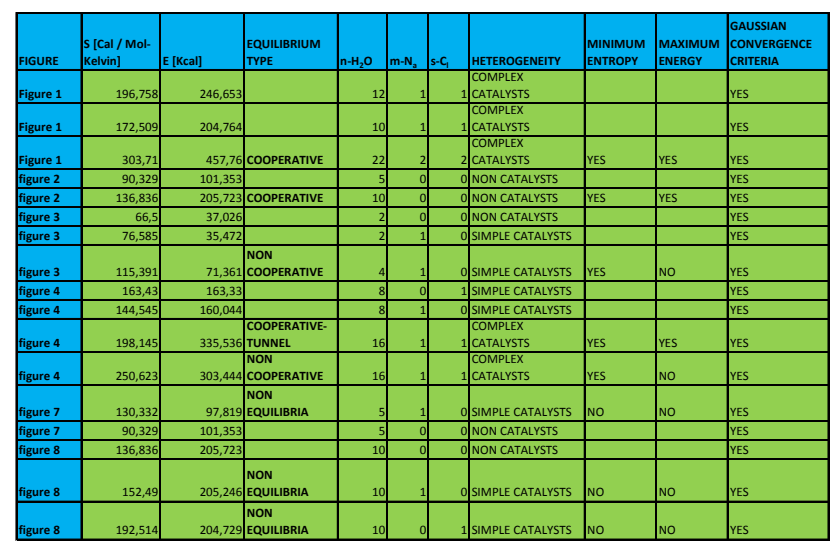

Figure 9. Equilibria Type as a function of Cooperation

\section{Conclusions}

- Each physical variable is represented by anHermitian operator whose norm allows us to obtain mixed strategy Nash's equilibrium in Hilbert space.

- By virtue of the theorems demonstrated in this paper, properties of Hermitian operators can only be used in symmetric games, which can be represented by complex matrix.

- Mixed strategy Nash's equilibrium in a bimatrix symmetric game represents a cooperative solution.

- The idea of isolated physicalsystems has its explanation, the simplification of variables and relationships among the parts. On the contrary, complex systems analyze entirety, synergy and interactions as the cause of a common objective denominated cooperation.

- The measurement process in quantum mechanics is the best example of strategic interaction between "human-subje ct and quantum-object."
Therefore, we have a minimum of two players when we carry out a measurement of a physical variable such us energy, speed, mo mentum.

- In physics, the existence of isolated systems with different unique objectives and disconnected to each other is untenable. This paper shows the necessity of introducing other elements like cooperation and optimality as foundations of the laws of quantum mechanics. We proved that Nash's equilibrium is the hidden optimal principle of quantum mechanics.

- The apparent ERP paradox is completely resolved by using revelation principle for strategic-form games, because it demonstrates that a correlated game is completely equivalent to a communication game.

Consequently, it is not necessary to speak of communication and worse even of information speed if a game is already correlated.

- Econophysics is both to use rational approaches of economics in the foundations of physics (in special, quantum mechanics) and to transpose physics formalism of quantum theory, statistical mechanics, electrodynamics and others in economics.

\section{REFERENCES}

[1] Aumann, R., "Correlated Equilibria as an Expression of Bayesian Rationality”,Econometrica, 55:1-18, 1987.

[2] Bar-Yam, Y.,"Dynamics of Complex Systems”, Addison-Wesley, Reading, Massachusetts, 1997.

[3] Boccara, N.,"Modeling Complex Sy stems", Springer-Verlag, Heidelberg, 2004.

[4] Bouwmeester, D., Eckert, A.,Zeilinger, A.,"The Physics of Quantum Information”, Springer-Verlag, London, UK, 2001.

[5] DrorShafiret all. Resolving the time when an electron exist a tunneling barrier. Nature, Letter. 343-346, 2012.

[6] Einstein, A.,Podolsky, B., Rosen, N., Phys. Rev. 47, 777, 1935.

[7] Hirvensalo, M.,"Quantum Computing”. Springer-Verlag, Berlin, 2001.

[8] Jiménez, E.,"Quantum Games and Minimum Entropy”, Springer, Lecture Notes in Computer Science 2669, Canada, p 216-225, 2003.

[9] Jiménez, E., “Quantum Games: Mixed Strategy Nash's Equilibrium Represents Minimum Entropy", Journal of Entropy, Vol 5, Issue4, p 313-347, 2003.

[10] Jiménez, E., Moya, D.,"Econophysics: From Game Theory and Information Theory to Quantum Mechanics",Physica A, 348C, p 505-543, 2005.

[11] Jiménez, E., "Econophysics Applied to Oil, Banking, Experimental Economics and Business Intelligence", EPPetroecuador, p 1-340, 2009. 
[12] Machiavello, C., Palma, G., Zeilinger, A.,"Quantum Computation and Quantum Information Theory", World Scientific, London, UK, 2000.

[13] Meyer, P.,"Quantum Probability for Probabilities”, Lecture Notes in Mathematics 1538. Springer-Verlag, Berlin, 1995.

[14] Myerson, R.,"Game Theory Analysis of Conflict”, Massachusetts, 1991.

[15] Morrone $\mathbf{J}$ et all. The Journal of Chemical Physics 130. 204511. 2009.
[16] Shiryayev, A.,"Probability", Springer-Verlag, New York, 1984.

[17] S. Suppiahet all. Clean hydrogen production with the $\mathrm{Cu}-\mathrm{Cl}$ cycle-Progress of international consortium, I: Experimental unit operations. International Journal of Hydrogen Energy. Vol 36, Iss 24. Pgs 15472-15485. 2012.

[18] Qiang Shao et all. From protein denaturant to protectant: Comparative molecular dynamics study of alcohol/ protein interactions. AIP BioChemical Physics, 2012. 